\title{
Grain Boundary Space Charge Effect in Zirconia
}

\section{Experimental Evidence}

\author{
Xin Guo ${ }^{a, b, z}$ and Yong Ding ${ }^{\mathrm{c}}$ \\ ${ }^{a}$ Elektrokeramische Materialien, and ${ }^{c}$ Mikrostrukturforschung, Institut für Festkörperforschung, \\ Forschungszentrum Jülich, 52425 Jülich, Germany \\ ${ }^{b}$ Max-Planck-Institut für Festkörperforschung, 70569 Stuttgart, Germany
}

According to the space charge concept, oxygen vacancies are depleted in the grain boundary space charge layers of acceptor-doped $\mathrm{ZrO}_{2}$; whereas electrons are expected to accumulate in the space charge layers if present, leading to significant changes in the grain boundary electrical properties. In this work, electrons were introduced to 8 mol $\% \mathrm{TiO}_{2}$ and 7.36 mol $\% \mathrm{Y}_{2} \mathrm{O}_{3}$-codoped $\mathrm{ZrO}_{2}$ by annealing in the mixture of $2 \% \mathrm{H}_{2}-\mathrm{Ar}$; as a result, the grain boundary arc disappeared, and the electrical conductivities increased considerably, especially for the sample with finer grain size. Both phenomena suggest highly conductive grain boundaries resulting from the accumulation of electrons in the space charge layers.

(C) 2003 The Electrochemical Society. [DOI: 10.1149/1.1625948] All rights reserved.

Manuscript submitted November 18, 2002; revised manuscript received March 18, 2003. Available electronically December 9 2003.

The so-called brick layer model is commonly used to explain the electrical properties of $\mathrm{ZrO}_{2}$ ceramics; as illustrated in Fig. 1a, cubic grains of the same size and homogeneous grain boundaries are assumed. The grain boundaries of acceptor-doped $\mathrm{ZrO}_{2}$ are highly resistive to the grain-to-grain transfer of charge carriers, resulting in a grain boundary conductivity which is $c a$. two orders of magnitude lower than the bulk conductivity; ${ }^{1-18}$ an intergranular siliceous phase was suggested to account for the very low grain boundary conductivity. ${ }^{16}$ However, in materials of high purity in which the siliceous phase was not observed, the specific grain boundary conductivity was still $c a$. two orders of magnitude lower than that of the bulk, ${ }^{3,17}$ indicating additional causes for the low grain boundary conductivity.

According to the core-space charge layer model, ${ }^{19}$ a grain boundary consists of a grain boundary core (crystallographic mismatch zone) and two adjacent space charge layers. In 2 mol \% $\mathrm{Y}_{2} \mathrm{O}_{3}$-doped $\mathrm{ZrO}_{2}$, the enrichments at the grain boundaries of additionally added divalent and trivalent minority solutes were found to be significant, whereas enrichments of tetravalent and pentavalent minority solutes were not observed, ${ }^{20}$ indicating positive space charge potential $\Delta \varphi(0)$, or negative space charge. The positively charged oxygen vacancies (charge carriers in doped $\mathrm{ZrO}_{2}$ ) are therefore depleted in the space charge layers as illustrated in Fig. 1b. In materials of high purity, the depletion of oxygen vacancies has also been suggested to be responsible for the very low grain boundary conductivity. ${ }^{3,21-26}$

However, the Debye length of $\mathrm{ZrO}_{2}$ with a typical dopant level appears to be as small as $0.1 \mathrm{~nm}$. Such a small thickness makes the role of the space charge layers questionable. But the dopant concentration is frozen at temperatures $<1000^{\circ} \mathrm{C}$, the dopant profile is, to a first approximation, expected to be constant with regard to the local coordinate. In such a Mott-Schottky situation, ${ }^{27}$ the screening effect is reduced, leading to an effective thickness which depends on the dopant segregation and its value can be distinctly greater than the Debye length; indeed the actual thickness of the space charge layer calculated from impedance spectroscopy is larger than the Debye length. The grain boundary thickness $\delta_{\mathrm{gb}}$ was determined to be $\sim 4.8$ $\mathrm{nm}$ for $8 \mathrm{~mol} \% \mathrm{Y}_{2} \mathrm{O}_{3}$-doped $\mathrm{ZrO}_{2},{ }^{25,26} \sim 5.4 \mathrm{~nm}$ for $8.2 \mathrm{~mol} \%$ $\mathrm{Y}_{2} \mathrm{O}_{3}$-doped $\mathrm{ZrO}_{2},{ }^{3}$ and $\sim 5.0 \mathrm{~nm}$ for $2 \mathrm{~mol} \% \mathrm{Y}_{2} \mathrm{O}_{3}$-doped $\mathrm{ZrO}_{2},{ }^{28}$ being independent of grain size. The $\delta_{\mathrm{gb}}$ values agreed well with the yttrium segregation, which was determined to be confined within a width of $4-6 \mathrm{~nm}$ across the grain boundary core. ${ }^{29,30}$ Because the thickness of the grain boundary core (crystallographic mismatch zone) is usually $\leqslant 1 \mathrm{~nm},{ }^{29,30}$ the yttrium segregation should mostly occur in the space charge layers; such a phenomenon is a good

z E-mail: x.guo@fz-juelich.de indicator of the negative space charge, owing to the negative effective charge of yttrium in the zirconium lattice $\left(Y_{\mathrm{Zr}}^{\prime}\right)$. As usual composition measurement techniques cannot distinguish between free and associated defects, the yttrium profile across a grain boundary measured by electron energy loss spectroscopy (EELS) ${ }^{29}$ also suggests a space charge situation; the seemingly low value (the yttrium concentration at the grain boundary is higher than that in the bulk by a factor of $\sim 2$ ) must actually correspond to a much higher space charge effect, because in the bulk most of the yttrium is essentially associated. The thickness of a space charge layer (roughly one half of the grain boundary thickness) is thus $\sim 2.5 \mathrm{~nm}$, which can be increased by the addition of low-valence cations strongly segregating to the grain boundaries, e.g., $\mathrm{Al}^{3+} \cdot{ }^{26}$

In 1982, Burggraaf et al. ${ }^{3}$ proposed that the depletion of oxygen vacancies in the grain boundary space charge layers is (at least partly) responsible for the very low grain boundary conductivity. Later, Heyne ${ }^{21}$ developed a model on the basis of the oxygen vacancy depletion in the space charge layers, but it predicted much too high an activation energy for the grain boundary conductivity; defect association was suggested to account for the discrepancy. Based on the space charge formalism by Kliewer and Koehler, ${ }^{31}$ Bingham et al. ${ }^{23}$ numerically calculated the grain boundary resistivity resulting from the depletion of oxygen vacancies for $3 \mathrm{~mol} \%$ $\mathrm{Y}_{2} \mathrm{O}_{3}$-doped $\mathrm{ZrO}_{2}$, which was 50 to 120 times higher than the bulk resistivity, agreeing well with experiments. More recently, $\mathrm{Y}_{2} \mathrm{O}_{3}$-doped $\mathrm{ZrO}_{2}$ of high purity was studied, and a Schottky barrier model $^{25}$ was proposed. According to the study, ${ }^{25}(i)$ the grain boundary conductivity is $c a$. two orders of magnitude lower than the bulk conductivity, accountable by the oxygen vacancy depletion in the space charge layers, and ( $i i)$ the activation energy for the grain boundary conductivity is determined by the space charge potential.

The above space charge concept is supported by two phenomena: (i) after quenching from high temperatures, subsequent annealing at temperatures lower than $800^{\circ} \mathrm{C}$, below which the relocation of the intergranular siliceous phase is impossible, decreased the grain boundary conductivity. ${ }^{15}$ This phenomenon can only be explained by the redistribution of oxygen vacancies in the space charge layers, as oxygen vacancies, in contrast to cations, still have very good mobility at these temperatures; (ii) it is known that $\mathrm{Al}_{2} \mathrm{O}_{3}$ can effectively scavenge the siliceous phase from the grain boundaries, ${ }^{26,32-34}$ but the $\mathrm{Al}_{2} \mathrm{O}_{3}$ addition actually decreased the grain boundary conductivity when the addition was below the solubility, ${ }^{26,34,35}$ contrary to the expected conductivity increase. This phenomenon can be explained by the increase of the space charge potential, thereby decrease of the oxygen vacancy concentration in the space charge layers. The increase of the space charge potential most probably resulted from the strong aluminum segregation to the grain boundaries. ${ }^{26}$ 
(b)

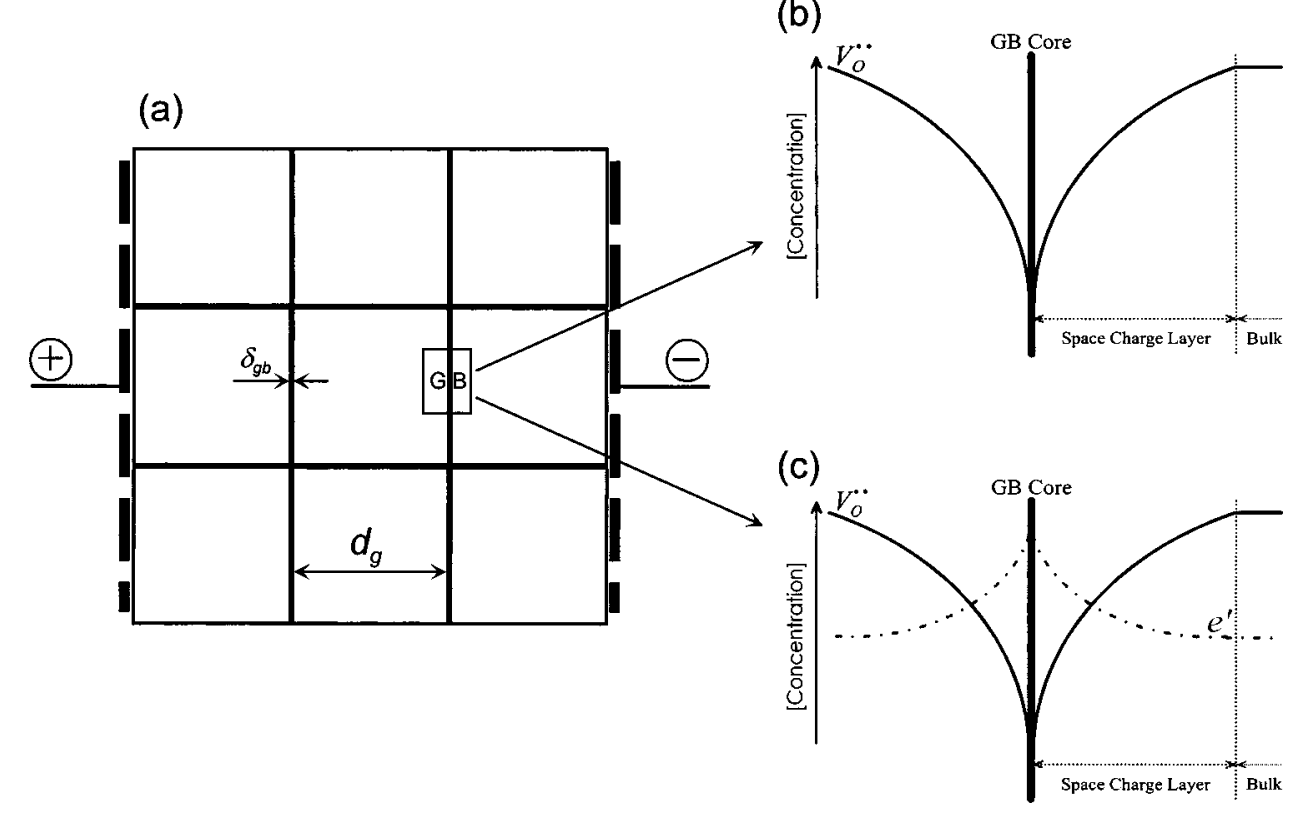

Figure 1. Schematic representations of (a) brick layer model, (b) and (c) charge carrier distributions in the grain boundary space charge layers.

However, if the space charge concept is correct, electrons, as a result of the negative charge, should accumulate in the space charge layers if present, as illustrated in Fig. 1c. A numerical simulation ${ }^{36}$ of the $\mathrm{SrTiO}_{3}$ grain boundary revealed such an electron accumulation. The accumulation of electrons in the space charge layers can be of importance for low oxygen partial pressures, high temperatures, and high space charge potentials.

Considerable electrons can be introduced to acceptor-doped $\mathrm{ZrO}_{2}$ by annealing at high enough temperatures and low enough oxygen partial pressures (e.g., $T=1300^{\circ} \mathrm{C}$ and $P_{\mathrm{O}_{2}}=10^{-35}$ $\mathrm{atm}^{37}$ ), but such extreme conditions are experimentally difficult to achieve; the difficulty can be overcome by the addition of metal ions with different valence states, in this aspect $\mathrm{TiO}_{2}$ is a good example. ${ }^{38-43}$ When dissolved, $\mathrm{Ti}^{4+}$ substitutionally replaces $\mathrm{Zr}^{4+}$ in the $\mathrm{ZrO}_{2}$ fluorite lattice; ${ }^{38}$ and $\mathrm{TiO}_{2}$ can be dissolved in $\mathrm{ZrO}_{2}$ in a large amount, e.g., $15 \mathrm{~mol} \%$ in $12 \mathrm{~mol} \% \mathrm{Y}_{2} \mathrm{O}_{3}$-doped $\mathrm{ZrO}_{2},{ }^{38} 13$ mol \% in $3 \mathrm{~mol} \% \mathrm{Y}_{2} \mathrm{O}_{3}$-doped $\mathrm{ZrO}_{2},{ }^{43}$ and $10-15 \mathrm{~mol} \%$ in $8 \mathrm{~mol}$ $\% \mathrm{Y}_{2} \mathrm{O}_{3}$-doped $\mathrm{ZrO}_{2}$, ${ }^{41}$ without changing the original crystal structure. While annealing at low oxygen partial pressures, titanium ions $\left(\mathrm{Ti}^{4+}\right)$ is reduced to give electrons, such a reaction in Kröger-Vink notation is ${ }^{40}$

$$
\mathrm{O}_{\mathrm{O}}^{\times} \rightarrow \mathrm{V}_{\mathrm{O}}^{\bullet}+2 \mathrm{e}^{\prime}+1 / 2 \mathrm{O}_{2}
$$

In this work, $\mathrm{TiO}_{2}$ and $\mathrm{Y}_{2} \mathrm{O}_{3}$-codoped $\mathrm{ZrO}_{2}$ samples of high purity were sintered at different temperatures for different hours to achieve very different grain sizes. The samples were initially annealed in pure oxygen to ensure purely ionic conduction, then electrons were introduced by annealing in an atmosphere with a very low oxygen partial pressure (mixture of $2 \%$ hydrogen and argon). By comparing the impedance spectra and the electrical conductivities obtained for the cases of high and low oxygen partial pressures, one can elucidate whether electrons can accumulate in the space charge layers as expected, which is a crucial test to the space charge concept.

\section{Experimental}

$\mathrm{ZrO}_{2}$ powder (quoted $\mathrm{SiO}_{2}$ content $\sim 50$ ppm by weight) doped with $8 \mathrm{~mol} \% \mathrm{Y}_{2} \mathrm{O}_{3}\left(8 \mathrm{Y}-\mathrm{ZrO}_{2}\right)$ purchased from Tosoh-Zirconia (Tokyo, Japan), and $\mathrm{TiO}_{2}$ powder with a purity of $99.99 \%$ purchased from CERAC (Wisconsin, USA) were mixed to prepare powder with a composition of $8 \mathrm{~mol} \% \mathrm{TiO}_{2}-92 \mathrm{~mol} \%\left(8 \mathrm{Y}_{-}-\mathrm{ZrO}_{2}\right)$; the actual composition was then $8 \mathrm{~mol} \% \mathrm{TiO}_{2}-7.36 \mathrm{~mol} \% \mathrm{Y}_{2} \mathrm{O}_{3}-84.64$ mol $\% \mathrm{ZrO}_{2}$. In order to obtain highly dense ceramic samples, the powder was cold-isostatically pressed at $1000 \mathrm{MPa}$ into pellets, then sintered first at $1400^{\circ} \mathrm{C}$ in air for $2 \mathrm{~h}$ to get a fine grain size; afterwards, the pellet was cut into two samples, one of which was further fired at $1700^{\circ} \mathrm{C}$ for $10 \mathrm{~h}$ to get a coarse grain size. Except for the very different grain sizes, the two samples were thereby almost identical. The relative densities of the sintered pellets were all above 96\% (Table I). The added $\mathrm{TiO}_{2}$ amount was well below its solubility limit in $\mathrm{ZrO}_{2}$, the phase was therefore purely cubic, as confirmed by X-ray diffraction (XRD, see Fig. 2a).

The microstructure was investigated by means of scanning electron microscopy (SEM, type Stereoscan 420), and high resolution transmission electron microscopy (HRTEM, Philips CM20 ST, operating at $200 \mathrm{KV}$ ). The SEM investigations were carried out on polished and thermally etched surfaces, and average grain sizes $\left(d_{\mathrm{g}}\right)$ were determined (see Table I). The HRTEM samples were prepared by standard methods, involving mechanical grinding to a thickness of about $0.1 \mathrm{~mm}$, dimpling to about $10 \mu \mathrm{m}$, then ion-beam milling to electron transparency.

The impedance spectra were recorded in the temperature range of 325 to $550^{\circ} \mathrm{C}$, and over the frequency range of 0.1 to $10^{6} \mathrm{~Hz}$ at a amplitude of $100 \mathrm{mV}$ with a Solartron 1260 frequency response analyzer. The temperature fluctuation at each temperature point was controlled to be within $\pm 0.1^{\circ} \mathrm{C}$. Painted platinum electrodes were used, the paint was applied to the entire areas of both sides of the pellets and fired at $1100^{\circ} \mathrm{C}$ for $1 \mathrm{~h}$. Oxygen with a purity of

Table I. Sintering condition (temperature and time), relative density, average grain size $\left(d_{\mathrm{g}}\right)$, and activation energies for the electrical conductivities measured in $\mathrm{O}_{2}\left(E_{\mathrm{a}}^{\mathrm{bulk}}\right.$ and $\left.E_{\mathrm{a}}^{\mathrm{gb}}\right)$ and in $2 \% \mathrm{H}_{2}-\mathrm{Ar}\left(E_{\mathrm{a}}\right)$, respectively.

\begin{tabular}{cccccc}
$\begin{array}{c}\text { Sintering } \\
\text { condition }\end{array}$ & $\begin{array}{c}\text { Density } \\
(\%)\end{array}$ & $\begin{array}{c}d_{\mathrm{g}} \\
(\mu \mathrm{m})\end{array}$ & $\begin{array}{c}E_{\mathrm{a}}^{\text {bulk }} \\
\text { in } \mathrm{O}_{2} \\
(\mathrm{eV})\end{array}$ & $\begin{array}{c}E_{\mathrm{a}}^{\mathrm{gb}} \\
\text { in } \mathrm{O}_{2} \\
(\mathrm{eV})\end{array}$ & $\begin{array}{c}E_{\mathrm{a}} \\
\text { in } 2 \% \mathrm{H}_{2}-\mathrm{Ar} \\
(\mathrm{eV})\end{array}$ \\
\hline $1400^{\circ} \mathrm{C} \times 2 \mathrm{~h}$ & 96.4 & 1.6 & 1.00 & 1.11 & 0.73 \\
$1700^{\circ} \mathrm{C} \times 10 \mathrm{~h}$ & 98.3 & 28.6 & 1.00 & 1.14 & 0.76
\end{tabular}




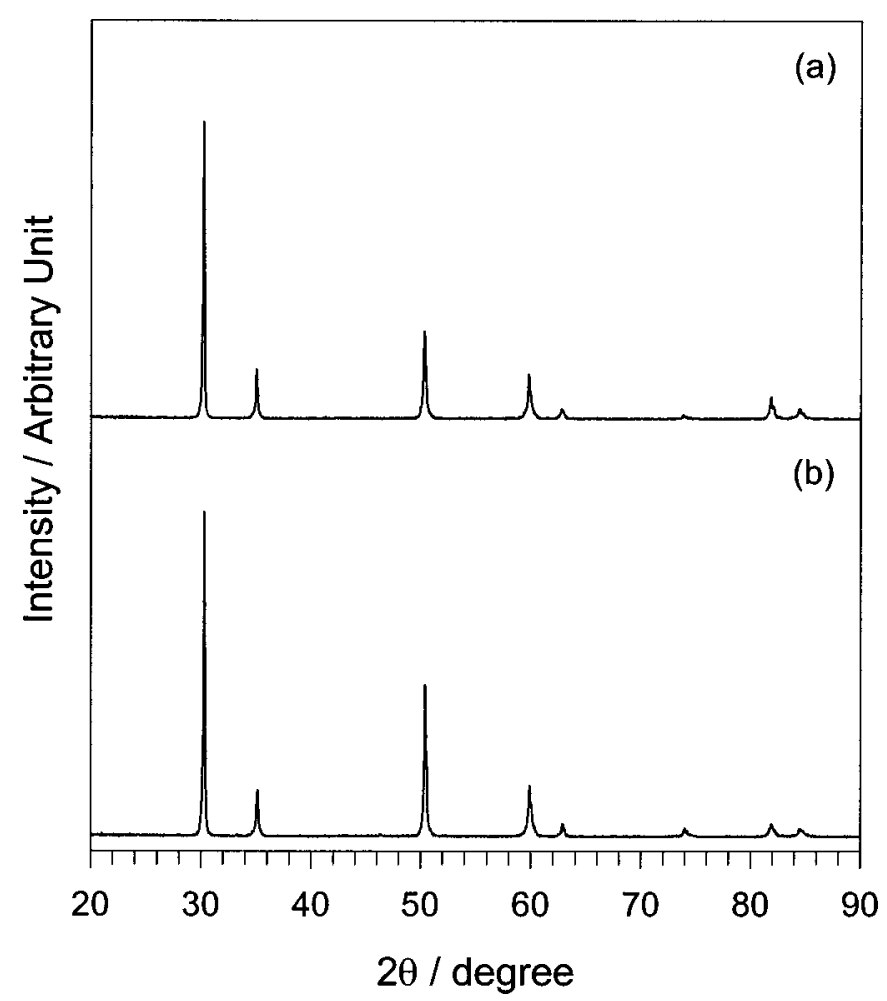

Figure 2. XRD patterns of the sample with an average grain size of 28.6 $\mu \mathrm{m}$, (a) as sintered and (b) after annealing in $2 \% \mathrm{H}_{2}$-Ar mixture at $950^{\circ} \mathrm{C}$ for $20 \mathrm{~h}$.

$99.9999 \%$, and a mixture of $2 \%$ hydrogen and argon was used to create different oxygen partial pressures, which were monitored by an oxygen sensor.

Typical impedance spectra in oxygen are shown in Fig. 3; in the order of decreasing frequency, three arcs corresponding to the responses of the bulk, the grain boundaries and the electrodes, respectively, were recorded. If not otherwise indicated, an equivalent circuit consisting of three $R Q$ circuits in series was used to interpret the impedance spectra. Here $R$ represents a resistance, $Q$ a constant phase element, characterized by two parameters, $C_{\mathrm{Q}}$ and $n$; corre-

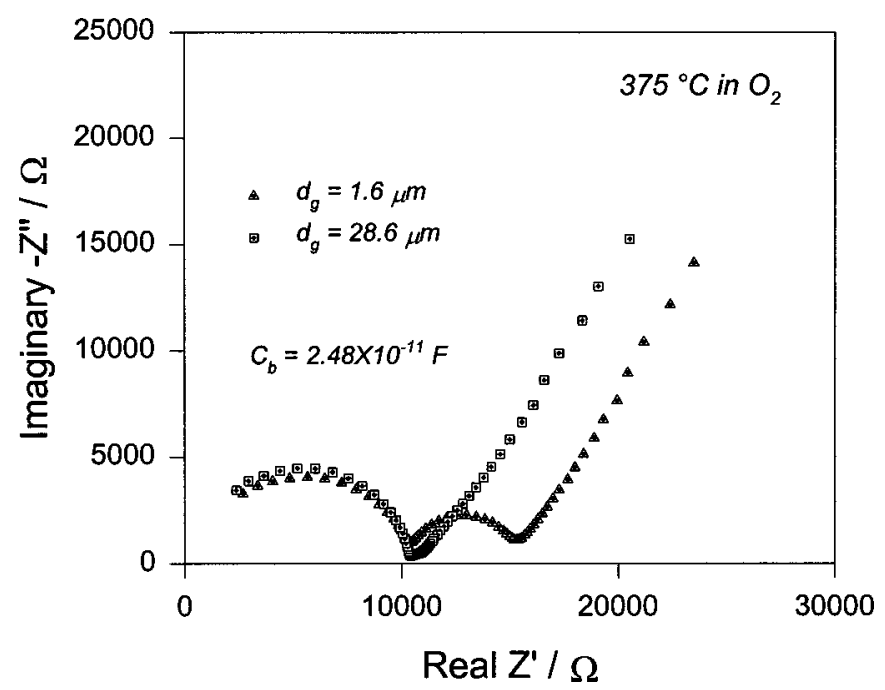

Figure 3. Impedance spectra at $375^{\circ} \mathrm{C}$ in oxygen for the samples with an average grain size of 1.6 and $28.6 \mu \mathrm{m}$, respectively. The spectra were normalized to a dimension of $\phi 10 \times 1 \mathrm{~mm}$.
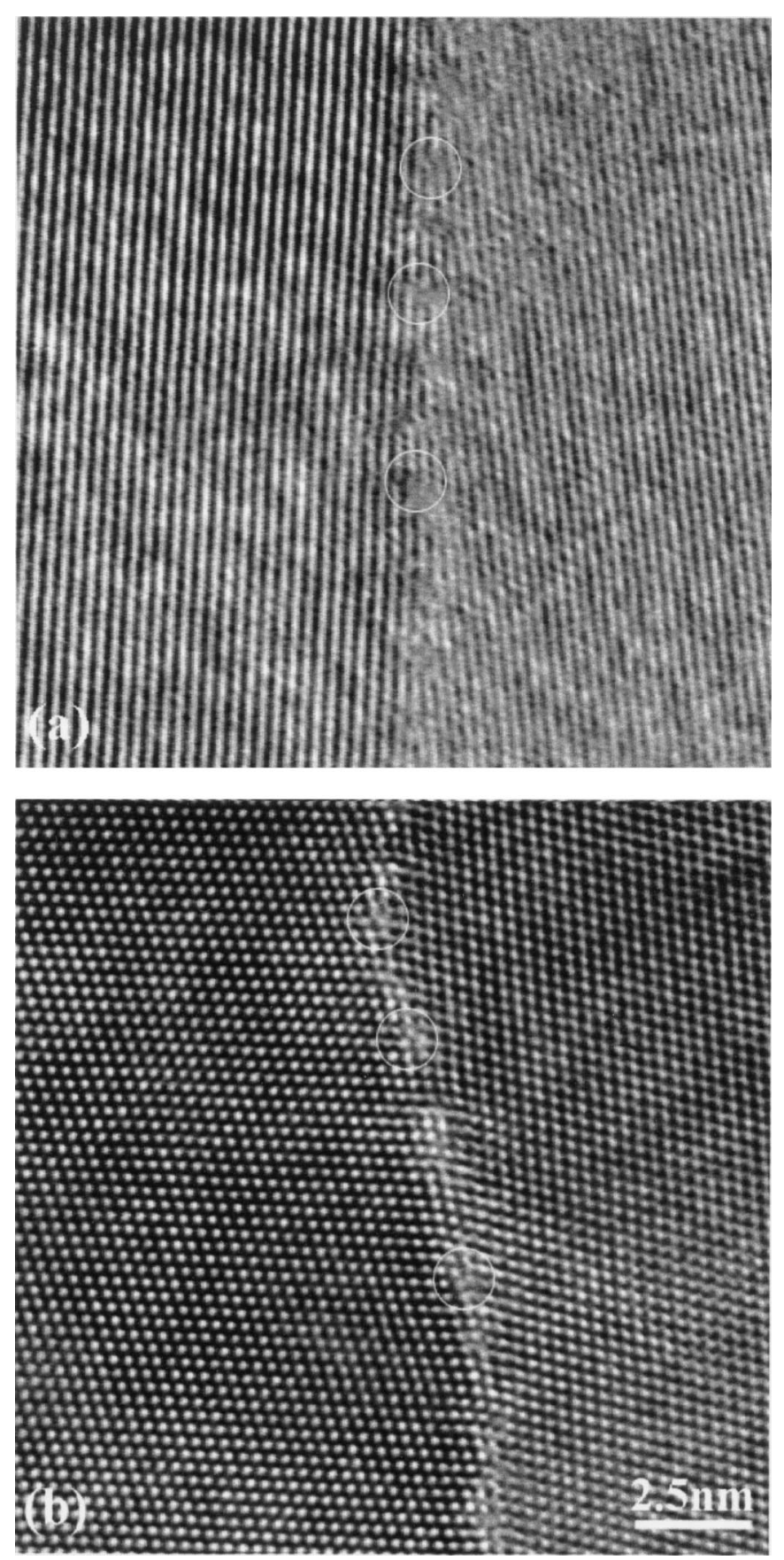

Figure 4. HRTEM micrographs of the sample with an average grain size of $28.6 \mu \mathrm{m}$, (a) as sintered and (b) after annealing in $2 \% \mathrm{H}_{2}$-Ar mixture at $950^{\circ} \mathrm{C}$ for $20 \mathrm{~h}$. The misfit dislocations at the grain boundaries are highlighted by circles.

sponding capacitances $C$ can be calculated from $C=\left(R^{1-n} C_{\mathrm{Q}}\right)^{1 / n}$. The bulk resistance $\left(R_{\text {bulk }}\right)$ and capacitance $\left(C_{\text {bulk }}\right)$, and the grain boundary resistance $\left(R_{\mathrm{gb}}\right)$ and capacitance $\left(C_{\mathrm{gb}}\right)$ of the samples were obtained in this way.

\section{Results}

High purity powders were used to prepare the samples, the grain boundaries of the sintered samples were therefore essentially free of any second phase, as demonstrated by the HRTEM micrograph shown in Fig. 4a: direct grain-to-grain contacts were prevailing at the grain boundaries. It is noted that even the dislocations at the 
$\mathrm{T} /{ }^{\circ} \mathrm{C}$

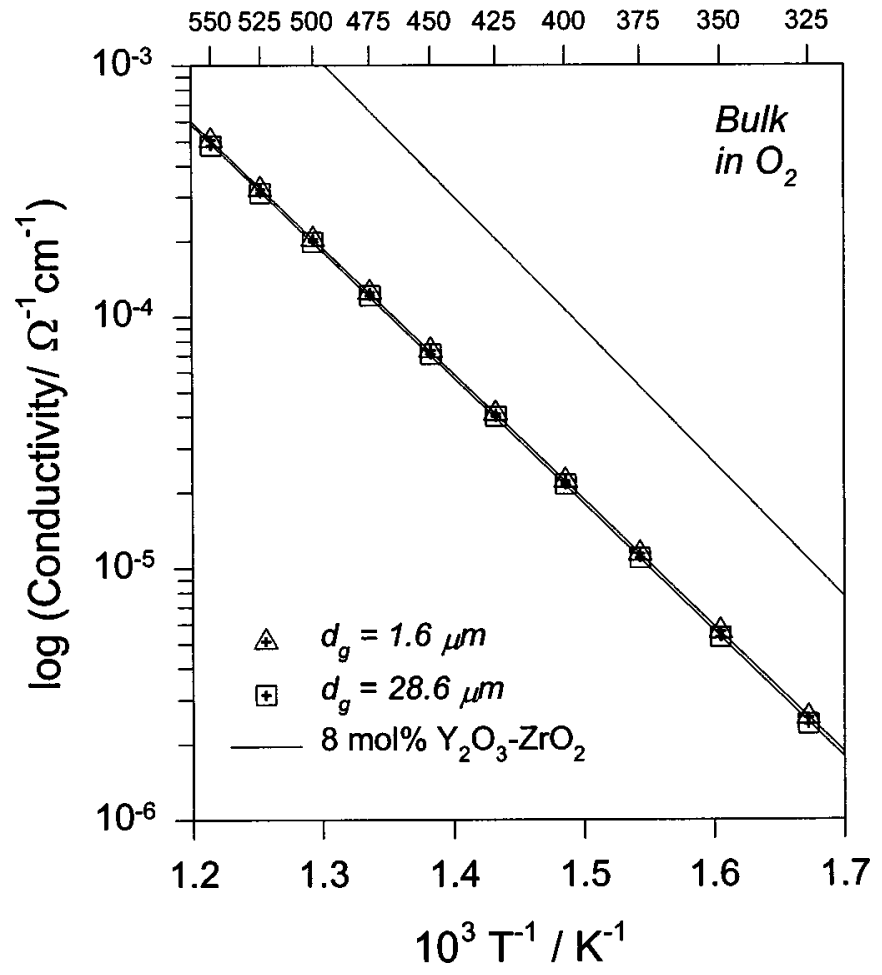

Figure 5. Temperature dependences of bulk conductivities measured in oxygen for the samples with an average grain size of 1.6 and $28.6 \mu \mathrm{m}$, respectively. The conductivity of $8 \mathrm{~mol} \% \mathrm{Y}_{2} \mathrm{O}_{3}$ doped $\mathrm{ZrO}_{2}$ is also plotted for comparison.

grain boundary were visible. The complexity of the siliceous phase was thus avoided, under such a condition the space charge layers are dominant at the grain boundaries. ${ }^{25}$

The temperature dependence of the bulk conductivity $\sigma_{\text {bulk }}$ and the specific grain boundary conductivity, $\sigma_{\mathrm{gb}}$, measured in oxygen are presented in Fig. 5 and 6; the specific grain boundary conductivity was roughly two orders of magnitude lower than the bulk conductivity. The conductivities of $8 \mathrm{~mol} \% \mathrm{Y}_{2} \mathrm{O}_{3}$-doped $\mathrm{ZrO}_{2}$ from a previous work ${ }^{25}$ are also plotted for comparison; it is obvious that the addition of $\mathrm{TiO}_{2}$ decreased both the bulk and the grain boundary conductivities, which has been extensively observed previously. ${ }^{39-43}$ The bulk conductivity was independent of grain size, but the grain boundary conductivity was higher for a finer grain size, suggesting that the grain boundary conductivity would be even higher when the grain size is in nanoscale, which has actually been observed for 2.9 mol \% $\mathrm{Y}_{2} \mathrm{O}_{3}$-doped $\mathrm{ZrO}_{2} .{ }^{44}$ Corresponding activation energies for the bulk and the specific grain boundary conductivities are given in Table I.

In Fig. 5 and 6, the bulk conductivity was calculated from $\sigma_{\text {bulk }}=L /\left(R_{\text {bulk }} A\right)$, where $R_{\text {bulk }}$ is the bulk resistance, $A$ is the cross-sectional area, and $L$ is the thickness of the samples; while the specific grain boundary conductivity $\sigma_{\mathrm{gb}}$ from $^{17}$

$$
\sigma_{\mathrm{gb}}=\frac{L}{R_{\mathrm{gb}} A} \frac{\delta_{\mathrm{gb}}}{d_{\mathrm{g}}}
$$

with $\delta_{\mathrm{gb}}$ being the grain boundary thickness, which can be calculated from the capacitance according to ${ }^{25}$

$$
\delta_{\mathrm{gb}}=d_{\mathrm{g}} C_{\mathrm{bulk}} / \mathrm{C}_{\mathrm{gb}}
$$

$\mathrm{T} /{ }^{\circ} \mathrm{C}$

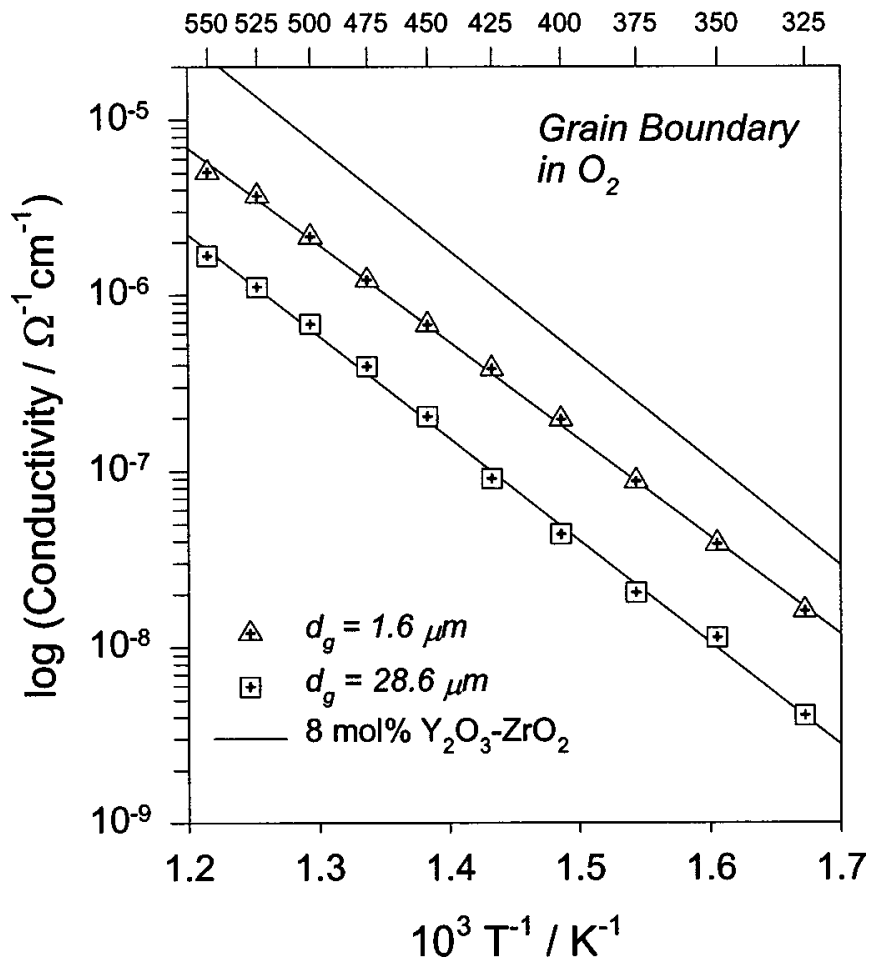

Figure 6. Temperature dependences of specific grain boundary conductivities measured in oxygen for the samples with an average grain size of 1.6 and $28.6 \mu \mathrm{m}$, respectively. The conductivity of $8 \mathrm{~mol} \% \mathrm{Y}_{2} \mathrm{O}_{3}$-doped $\mathrm{ZrO}_{2}$ is also plotted for comparison.

if approximating the dielectric constant of the grain boundary $\varepsilon_{\mathrm{gb}}$ by the bulk value $\varepsilon_{\text {bulk }}$. This approximation is not unreasonable in view of the fact that the dielectric constant of $\mathrm{ZrO}_{2}$ is quite composition insensitive. ${ }^{25,45}$ The validity of such an approximation has been experimentally proved for acceptor-doped $\mathrm{SrTiO}_{3}$, in which $\varepsilon_{\mathrm{gb}}$ was determined to be $\sim 0.95 \varepsilon_{\text {bulk }}$. ${ }^{46}$

Afterward, the samples were annealed at $950^{\circ} \mathrm{C}$ in a mixture of $2 \%$ hydrogen and argon for $20 \mathrm{~h}$. The oxygen diffusion coefficient $D$ in $8 \mathrm{~mol} \% \mathrm{TiO}_{2}-92 \mathrm{~mol} \%\left(8 \mathrm{Y}^{-} \mathrm{ZrO}_{2}\right)$ can be calculated from the Nernst-Einstein equation $D=\sigma k_{\mathrm{B}} T /\left(c z^{2}\right)$, which was $\sim 3.49$ $\times 10^{-6} \mathrm{~cm}^{2} \mathrm{~s}^{-1}$ at $950^{\circ} \mathrm{C}$; if estimating from this diffusion coefficient, $1 \mathrm{~h}$ is enough for a sample with a thickness of $1 \mathrm{~mm}$ to reach equilibrium with the atmosphere at $950^{\circ} \mathrm{C}$. In the Nernst-Einstein equation, $\sigma$ is the electrical conductivity, $k_{\mathrm{B}}$ is the Boltzmann constant, $T$ is the absolute temperature, $c$ is the concentration of charge carriers, and $z$ is the charge quantity. During annealing, the resistances of the samples at a few time points were also monitored to check whether the samples really reached equilibrium with the atmosphere. To give examples, after annealing in the mixture of $2 \%$ hydrogen and argon for $4.5 \mathrm{~h}$, the resistance of the sample with a grain size of $28.6 \mu \mathrm{m}$ was $8.177 \Omega$, the resistance decreased only a little bit to $7.937 \Omega$ after annealing for $15 \mathrm{~h}$, and it became $7.933 \Omega$ after annealing for $20 \mathrm{~h}$; for the sample with a grain size of $1.6 \mu \mathrm{m}$, the resistance was $8.460 \Omega$ after annealing for $2.5 \mathrm{~h}$, and it became $7.881 \Omega$ after annealing for $20 \mathrm{~h}$. It is thus clear that both samples reached equilibrium with the atmosphere after annealing for $20 \mathrm{~h}$.

The oxygen partial pressure of the $2 \% \mathrm{H}_{2}$ - $\mathrm{Ar}$ mixture at $950^{\circ} \mathrm{C}$ was measured by an oxygen sensor to be $\sim 1.7 \times 10^{-18}$ atm. According to Eq. 1, electrons were introduced to the samples during annealing. After annealing, the sample phase remained cubic (see Fig. 2b); no microstructure change was observed, and the grain boundaries remained free of any second phase (Fig. 4b). In Fig. 4b, 


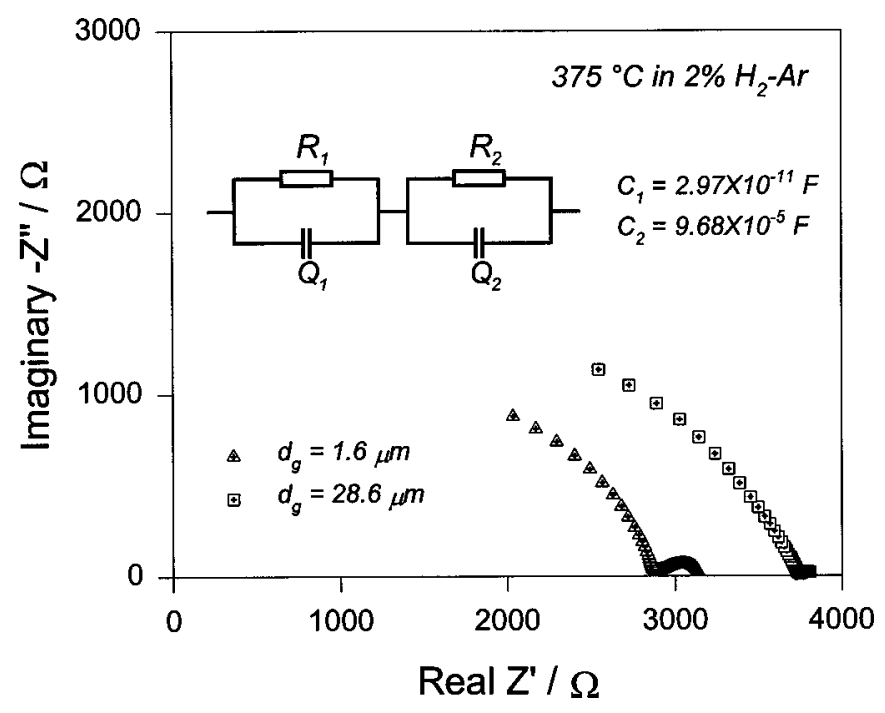

Figure 7. Impedance spectra at $375^{\circ} \mathrm{C}$ in $2 \% \mathrm{H}_{2}$ - $\mathrm{Ar}$ mixture for the samples with an average grain size of 1.6 and $28.6 \mu \mathrm{m}$, respectively. The spectra were normalized to a dimension of $\phi 10 \times 1 \mathrm{~mm}$.

the dislocations at the grain boundary are also clearly visible.

After the annealing at $950^{\circ} \mathrm{C}$, the samples were then cooled down to $550^{\circ} \mathrm{C}$ at a rate of $\sim 50^{\circ} \mathrm{C} / \mathrm{min}$ in the same atmosphere. The impedance spectra were then recorded at a temperature interval of $25^{\circ} \mathrm{C}$, beginning at $550^{\circ} \mathrm{C}$; typical spectra are shown in Fig. 7. Judging from the capacitance, the high frequency arc is bulk related, because its capacitance $\left(C_{1}\right.$ in Fig. 7$)$ agrees with the bulk value (see Fig. 3), and one order of magnitude higher than the apparatus (spectrometer, sample holder, leads etc.) capacitance $\left(\sim 10^{-12} \mathrm{~F}\right)$. The capacitance $C_{2}$ is more than three orders of magnitude larger than the grain boundary capacitance $C_{\mathrm{gb}}$ (e.g., $C_{\mathrm{gb}}=1.90 \times 10^{-8}$ $\mathrm{F}$ for the sample with an average grain size of $1.6 \mu \mathrm{m})$. It is thus obvious that the grain boundary arcs disappeared after the introduction of electrons.

The electrical conductivities calculated from $\sigma_{1}=L /\left(R_{1} A\right)$ are plotted in Fig. 8, the bulk conductivities measured in oxygen are also plotted for comparison. As indicated in Fig. 8, both conductivities measured in the $2 \% \mathrm{H}_{2}$ - $\mathrm{Ar}$ mixture were higher than the bulk conductivity measured in oxygen, this was due to the electronic conductivity; and the conductivity measured in the $2 \% \mathrm{H}_{2}$ - Ar mixture was higher for a finer grain size. Very similar phenomenon was also observed for $\mathrm{SrTiO}_{3} .{ }^{47}$

Corresponding activation energies for the conductivities measured in the $2 \% \mathrm{H}_{2}$-Ar mixture are also given in Table I, which are much smaller than the activation energies for the ionic conductivity, suggesting mixed conduction of oxygen vacancies and electrons. In $5 \mathrm{~mol} \% \mathrm{TiO}_{2}$ and $8 \mathrm{~mol} \% \mathrm{Y}_{2} \mathrm{O}_{3}$-codoped $\mathrm{ZrO}_{2}$ when $T=1000^{\circ} \mathrm{C}$ and $P_{\mathrm{O}_{2}}=10^{-18} \mathrm{~atm},{ }^{48}$ the electronic and the ionic partial conductivity were comparable, but with the ionic one being higher. As suggested by Fig. 8, a similar situation could be expected for the samples of this work, as well.

\section{Discussion}

As illustrated in Fig. 1a, in the current direction charge carriers can exclusively diffuse either parallel or perpendicular to the grain boundaries, so there are altogether two kinds of grain boundary resistances: the perpendicular resistance $R_{\mathrm{gb}}^{\perp}$, which is

$$
R_{\mathrm{gb}}^{\perp}=\frac{1}{\sigma_{\mathrm{gb}}^{\perp}} \frac{L}{A} \frac{\delta_{\mathrm{gb}}}{d_{\mathrm{g}}}
$$

and the parallel resistance $R_{\mathrm{gb}}^{\|}$, given by

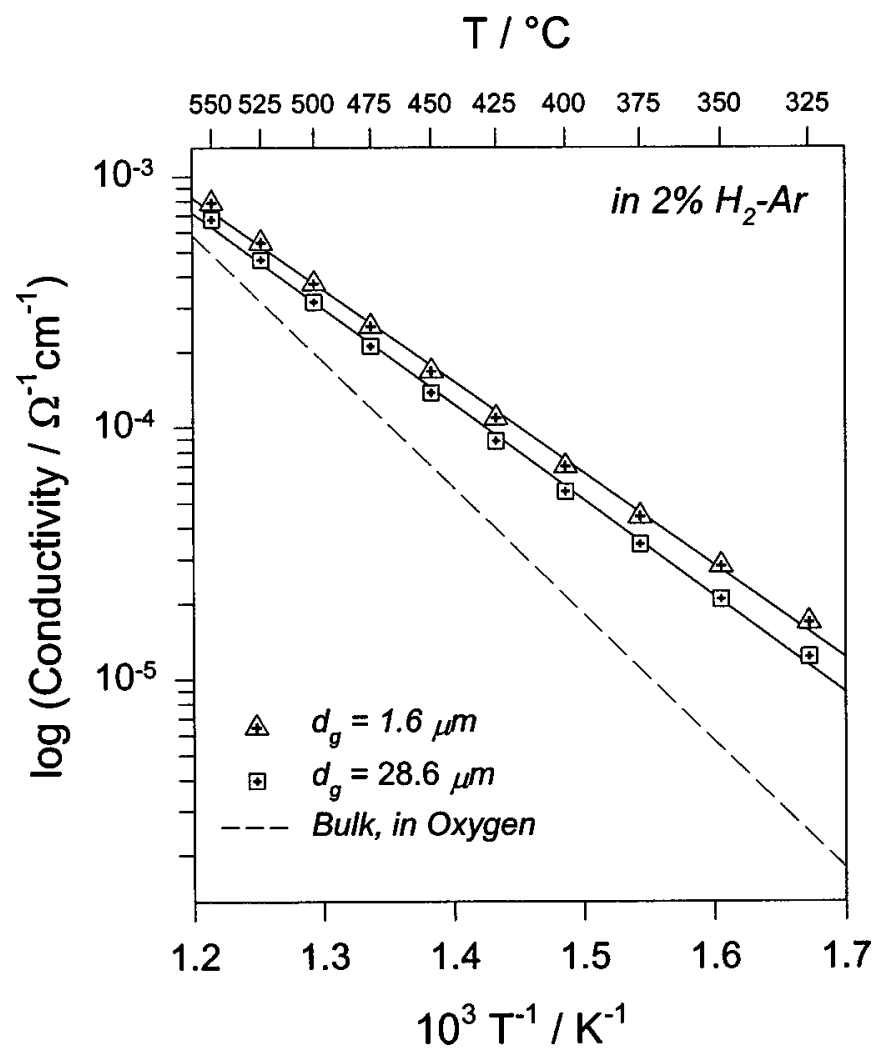

Figure 8. Temperature dependences of conductivities measured in $2 \%$ $\mathrm{H}_{2}$-Ar mixture for the samples with an average grain size of 1.6 and 28.6 $\mu \mathrm{m}$, respectively. The bulk conductivity measured in oxygen is also plotted for comparison.

$$
R_{\mathrm{gb}}^{\|}=\frac{1}{\sigma_{\mathrm{gb}}^{\|}} \frac{L}{A} \frac{d_{\mathrm{g}}}{\delta_{\mathrm{gb}}}
$$

Usually, the grain boundary conductivity perpendicular to and that parallel to the current direction are different, i.e., $\sigma_{\mathrm{gb}}^{\perp} \neq \sigma_{\mathrm{gb}}^{\|} \cdot{ }^{19}$ For materials with not very fine grain size $d_{\mathrm{g}} / \delta_{\mathrm{gb}} \sim 10^{3}$, it is obvious that $R_{\mathrm{gb}}^{\|} \gg R_{\mathrm{gb}}^{\perp}$; the parallel resistance $R_{\mathrm{gb}}^{\|}$is therefore usually neglected, the so-called grain boundary resistance $R_{\mathrm{gb}}$ is simply $R_{\mathrm{gb}}^{\perp}$. But, when taking the parallel resistance $R_{\mathrm{gb}}^{\|}$into consideration, the usual equivalent circuit becomes Fig. 9. A detailed analysis of the grain boundary impedance and conductance parallel and perpendicular to the current direction has been done in Ref. 19, but in this work we confine ourselves to resistances. The total resistance of a sample, $R_{\text {tot }}$, in this case is

$$
R_{\mathrm{tot}}=\frac{\left(R_{\mathrm{bulk}}+R_{\mathrm{gb}}^{\perp}\right) R_{\mathrm{gb}}^{\|}}{R_{\mathrm{bulk}}+R_{\mathrm{gb}}^{\perp}+R_{\mathrm{gb}}^{\|}}
$$

As demonstrated in Fig. 7, after annealing in the mixture of $2 \%$ $\mathrm{H}_{2}$-Ar, the grain boundary arc disappeared, i.e., $R_{\text {bulk }} \gg R_{\mathrm{gb}}^{\perp}$ and $R_{\mathrm{gb}}^{\|} \gg R_{\mathrm{gb}}^{\perp}$; the high frequency arc recorded under this condition represented the combination of the contributions from the bulk, and the grain boundaries parallel to the current direction, so corresponding capacitance was therefore comparable with the bulk capacitance. If the high frequency arc were solely due to the bulk, the conductivity would be independent of grain size as shown in Fig. 5; however, it was not the case as clearly demonstrated in Fig. 8. Now, $R_{\text {tot }}$ is simply 


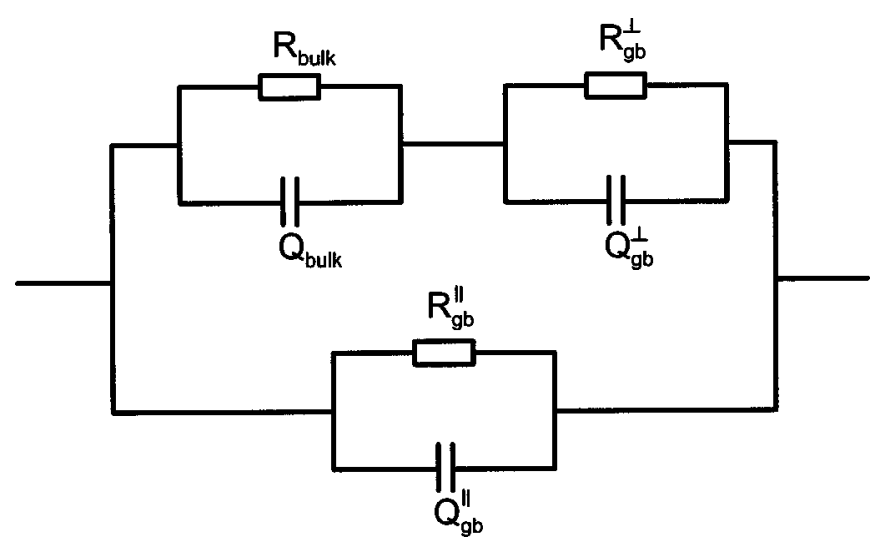

Figure 9. Equivalent electrical circuit if taking the parallel grain boundary resistance into consideration. In this figure, $R_{\text {bulk }}$ and $Q_{\text {bulk }}$ are the resistance and the constant phase element of the bulk, respectively; $R_{\mathrm{gb}}^{\perp}$ and $Q_{\mathrm{gb}}^{\perp}$ the resistance and the constant phase element of the perpendicular grain boundaries, respectively; and $R_{\mathrm{gb}}^{\|}$and $Q_{\mathrm{gb}}^{\|}$the resistance and the constant phase element of the parallel grain boundaries, respectively.

$$
R_{\mathrm{tot}}=R_{1}=\frac{R_{\mathrm{bulk}} R_{\mathrm{gb}}^{\|}}{R_{\mathrm{bulk}}+R_{\mathrm{gb}}^{\|}}
$$

with $R_{1}$ being the resistance of the high frequency arc shown in Fig. 7. Corresponding conductivity, $\sigma_{\text {tot }}$, is

$$
\frac{\sigma_{1}}{\sigma_{\text {bulk }}}=1+\frac{\sigma_{\mathrm{gb}}^{\|}}{\sigma_{\text {bulk }}} \frac{\delta_{\mathrm{gb}}}{d_{\mathrm{g}}}
$$

Note that at $325^{\circ} \mathrm{C}$ the conductivity of the sample with an average grain size of $1.6 \mu \mathrm{m}$ was larger than that of the sample with an average grain size of $28.6 \mu \mathrm{m}$ by $\sim 100 \%$; as $\delta_{\mathrm{gb}} / d_{\mathrm{g}} \approx 10^{-3}$, such an increase in conductivity requires that $\sigma_{\mathrm{gb}}^{\|} / \sigma_{\text {bulk }} \approx 10^{3}$, i.e., the grain boundaries parallel to the current direction were much more conductive than the bulk. The introduction of electrons simultaneously increased the electronic partial conductivity and the total conductivity both in the bulk and at the grain boundaries, however, the highly conductive grain boundaries suggests a more drastic increase in the grain boundary electronic partial conductivity, which must have been due to the accumulation of electrons in the space charge layers.

The ionic and the electronic conduction rails in the bulk and at the grain boundaries can now be described by Fig. 10. Note that the ionic and the electronic conduction took place on separate rails, any switch between the ionic and electronic conduction is impossible both in the bulk and at the grain boundaries. Comparing Fig. 9 and 10 , one has

$$
\begin{aligned}
& R_{\text {bulk }}=\frac{R_{\text {bulk }}^{\text {ion }} R_{\text {bulk }}^{\text {eon }}}{R_{\text {bulk }}^{\text {ion }}+R_{\text {bulk }}^{\text {eon }}} \\
& R_{\mathrm{gb}}^{\perp}=\frac{R_{\mathrm{gb}}^{\perp, \text { ion }} R_{\mathrm{gb}}^{\perp, \text { eon }}}{R_{\mathrm{gb}}^{\perp, \text { ion }}+R_{\mathrm{gb}}^{\perp, \text { eon }}} \\
& R_{\mathrm{gb}}^{\|}=\frac{R_{\mathrm{gb}}^{\|, \text {ion }} R_{\mathrm{gb}}^{\|, \text {eon }}}{R_{\mathrm{gb}}^{\| \text {,ion }}+R_{\mathrm{gb}}^{\|, \text {eon }}}
\end{aligned}
$$

As a result of the depletion of oxygen vacancies and the accumulation of electrons in the space charge layers, $R_{\mathrm{gb}}^{\perp \text {,ion }} \gg R_{\mathrm{gb}}^{\perp \text {,eon }}$ and $R_{\mathrm{gb}}^{\|, \text {ion }} \gg R_{\mathrm{gb}}^{\|, \text {eon }}$; therefore, the ionic transports across and along the grain boundaries were bypassed by the electronic one. Owing to the negligible capacitance of the parallel grain boundaries, an $R Q$ cir-

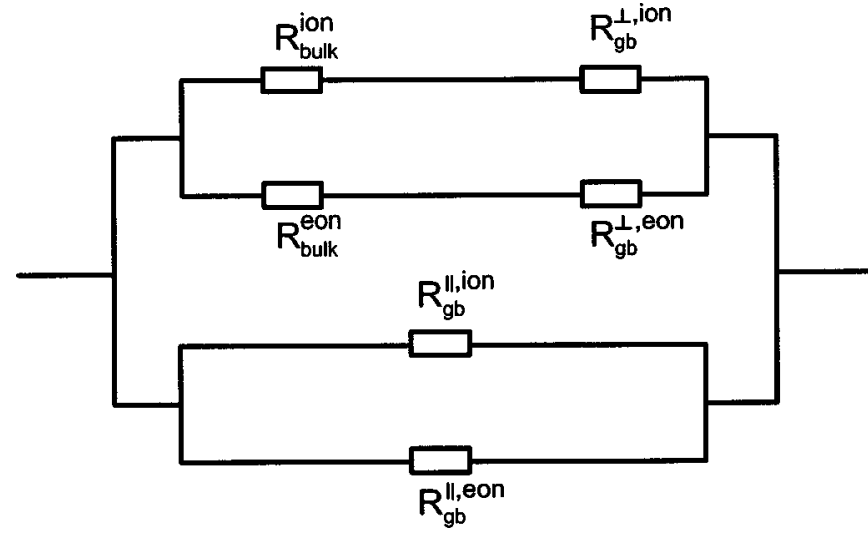

Figure 10. Ionic conduction rails and electronic conduction rails in the bulk and at the grain boundaries. In this figure, $R_{\text {bulk }}^{\text {ion }}$ is the ionic partial conductivity in the bulk, $R_{\text {bulk }}^{\text {eon }}$ is the electronic partial conductivity in the bulk; $R_{\mathrm{gb}}^{\perp \text {,ion }}$ the ionic partial conductivity at the perpendicular grain boundaries, $R_{\mathrm{gb}}^{\perp \text {,eon }}$ the electronic partial conductivity at the perpendicular grain boundaries; and $R_{\mathrm{gb}}^{\|, \text {ion }}$ the ionic partial conductivity at the parallel grain boundaries, $R_{\mathrm{gb}}^{\|, \text {eon }}$ the electronic partial conductivity at the parallel grain boundaries. Here the indices eon and ion refer to the electronic and the ionic charge carriers, respectively.

cuit with $Q$ being the constant phase element of the bulk and $R$ the parallel combination of the contributions from the bulk and the parallel grain boundaries is reasonably expected for the sample.

Acceptor-doped $\mathrm{CeO}_{2}$ is a mixed conductor of oxygen vacancies and electrons over a wide range of temperature and oxygen partial pressure, very similar accumulation of electrons in the space charge layers has also been proved for microcrystalline $e^{49}$ and nanocrystalline ${ }^{50} \mathrm{CeO}_{2}$.

\section{Conclusions}

Electrons were introduced to $8 \mathrm{~mol} \% \mathrm{TiO}_{2}$ and $7.36 \mathrm{~mol} \%$ $\mathrm{Y}_{2} \mathrm{O}_{3}$-codoped $\mathrm{ZrO}_{2}$ by annealing in a reducing atmosphere, and subsequently recorded impedance spectra and measured conductivities demonstrated that the grain boundary conductivity increased more drastically than the bulk one, and the grain boundaries became even more conductive than the bulk, suggesting the electron accumulation in the grain boundary space charge layers. It is thus clear that the charge carrier distribution (depletion of oxygen vacancies and accumulation of electrons) in the space charge layers is responsible for the grain boundary electrical properties of $\mathrm{ZrO}_{2}$.

\section{Acknowledgment}

Part of the work was done in the Max-Planck-Institut für Festkörperforschung, Stuttgart, Germany.

Forschungszentrum Jülich assisted in meeting the publication costs of this article.

\section{References}

1. N. M. Beekmans and L. Heyne, Electrochim. Acta, 21, 303 (1976).

2. T. van Dijk and A. J. Burggraaf, Phys. Status Solidi A, 63, 229 (1981).

3. M. J. Verkerk, B. J. Middelhuis, and A. J. Burggraaf, Solid State Ionics, 6, 159 (1982).

4. C. A. Leach, P. Tanev, and B. C. H. Steele, J. Mater. Sci. Lett., 5, 893 (1986)

5. S. P. S. Badwal and J. Drennan, J. Mater. Sci., 22, 3231 (1987).

6. A. el Barhmi, E. J. L. Schouler, A. Hammou, and M. Kleitz, Solid State Ionics, 28-30, 493 (1988).

7. S. P. S. Badwal and J. Drennan, J. Mater. Sci., 24, 88 (1989).

8. A. E. Hughes and B. A. Sexton, J. Mater. Sci., 24, 1057 (1989).

9. A. E. Hughes and S. P. S. Badwal, Solid State Ionics, 40/41, 312 (1990).

10. A. E. Hughes and S. P. S. Badwal, Solid State Ionics, 46, 265 (1991).

11. S. P. S. Badwal and J. Drennan, Solid State Ionics, 40/41, 869 (1990).

12. S. P. S. Badwal, F. T. Ciacchi, M. V. Swain, and V. Zelizko, J. Am. Ceram. Soc., 73, 2505 (1990).

13. S. P. S. Badwal and A. E. Hughes, J. Eur. Ceram. Soc., 10, 115 (1992). 
14. S. P. S. Badwal and S. Rajendran, Solid State Ionics, 70/71, 83 (1994).

15. S. P. S. Badwal, Solid State Ionics, 76, 67 (1995).

16. M. Gödickemeier, B. Michel, A. Orliukas, P. Bohac, K. Sasaki, L. Gauckler, H. Heinrich, P. Schwander, G. Kostorz, H. Hofmann, and O. Frei, J. Mater. Res., 9, 1228 (1994)

17. M. Aoki, Y.-M. Chiang, I. Kosacki, J. R. Lee, H. L. Tuller, and Y.-P. Liu, J. Am. Ceram. Soc., 79, 1169 (1996).

18. Y. Arachi, H. Sakai, O. Yamamoto, Y. Takeda, and N. Imanishai, Solid State Ionics, 121, 133 (1999).

19. J. Maier, Ber. Bunsenges. Phys. Chem., 90, 26 (1986).

20. S. L. Hwang and I. W. Chen, J. Am. Ceram. Soc., 73, 3269 (1990).

21. L. Heyne, in Mass Transport in Solids, E. Beniere and C. R. A. Catlow, Editors, p. 425, Plenum Press, New York (1983).

22. A. J. Burggraaf and A. J. A. Winnubst, in Surface and Near-Surface Chemistry of Oxide Materials, J. Nowotny and L.-C. Dufour, Editors, p. 449, Elsevier Science, Amsterdam (1988).

23. D. Bingham, P. W. Tasker, and A. N. Cormack, Philos. Mag. A, 60, 1 (1989).

24. X. Guo, Comput. Mater. Sci., 20, 168 (2001)

25. X. Guo and J. Maier, J. Electrochem. Soc., 148, E121 (2001)

26. X. Guo, W. Sigle, J. Fleig, and J. Maier, Solid State Ionics, 154-155, 555 (2002).

27. W. Schottky, Z. Phys., 113, 367 (1939).

28. J.-S. Lee and D.-Y. Kim, J. Mater. Res., 16, 2739 (2001)

29. Y. Ikuhara, P. Thavorniti, and T. Sakuma, Acta Mater, 45, 5275 (1997).

30. S. Stemmer, J. Vleugels, and O. van der Biest, J. Eur. Ceram. Soc., 18, 1565 (1998).

31. K. L. Kliewer and J. S. Koehler, Phys. Rev. A, 140, 1226 (1965).
32. E. P. Butler and J. Drennan, J. Am. Ceram. Soc., 65, 474 (1982).

33. J.-H. Lee, T. Mori, J.-G. Li, T. Ikegami, M. Komatsu, and H. Haneda, J. Am. Ceram. Soc., 83, 1273 (2000).

34. M. Miyayama, H. Yanagida, and A. Asada, Am. Ceram. Soc. Bull., 64, 660 (1985).

35. X. Guo, Phys. Status Solidi A, 183, 261 (2001).

36. R. Waser, Solid State Ionics, 75, 89 (1995).

37. T. H. Etsell and S. N. Flengas, Chem. Rev. (Washington, D.C.), 70, 339 (1970).

38. S. S. Liou and W. L. Worrell, Appl. Phys. A: Solids Surf., 49, 25 (1989).

39. H. Natio and H. Arashi, Solid State Ionics, 53-56, 436 (1992).

40. M. T. Colomer, L. S. M. Traqueia, J. R. Jurado, and F. M. B. Marques, Mater. Res. Bull., 30, 515 (1995).

41. L. S. M. Traqueia, T. Pagnier, and F. M. B. Marques, J. Eur. Ceram. Soc., 17, 1019 (1997).

42. M. T. Colomer, P. Duran, A. Caballero, and J. R. Jurado, Mater. Sci. Eng., A, 229, 114 (1997)

43. F. Capel, C. Moure, and P. Duran, J. Mater. Sci., 35, 345 (2000)

44. P. Mondal, A. Klein, W. Jaegermann, and H. Hahn, Solid State Ionics, 118, 331 (1999).

45. M. C. Steil, F. Thevenot, and M. Kleitz, J. Electrochem. Soc., 144, 390 (1997).

46. M. Vollman and R. Waser, J. Am. Ceram. Soc., 77, 235 (1994).

47. J. C. C. Abrantes, J. A. Labrincha, and J. R. Frade, J. Eur. Ceram. Soc., 22, 1683 (2002).

48. K. Kobayashi, Y. Kai, S. Yamaguchi, N. Fukatsu, T. Kawashima, and Y. Iguchi, Solid State Ionics, 93, 193 (1997).

49. X. Guo, W. Sigle, and J. Maier, J. Am. Ceram. Soc., 86, 77 (2003).

50. S. Kim and J. Maier, J. Electrochem. Soc., 149, J73 (2002). 